\title{
A randomised controlled demonstration trial of multifaceted nutritional intervention and or probiotics: the healthy mums and babies (HUMBA) trial
}

Karaponi Okesene-Gafa, ${ }^{1,2}$, Minglan Li ${ }^{1}$, Rennae S. Taylor ${ }^{1}$, John M. D. Thompson ${ }^{1}$, Caroline A. Crowther ${ }^{3}$, Christopher J. D. McKinlay ${ }^{3,4}$ and Lesley M. E. McCowan ${ }^{1,2^{*}}$

\begin{abstract}
Background: Maternal obesity is associated with adverse pregnancy outcomes and has lifelong negative implications for offspring health. The Institute of Medicine recommends limited gestational weight gain (GWG) in obese women for optimal maternal and infant outcomes. However, there is a gap regarding an effective and sustainable intervention strategy to achieve this goal. The aim of the healthy mums and babies (HUMBA) demonstration trial is to assess whether a multifaceted nutritional intervention and/or an oral probiotic treatment in obese pregnant women can reduce excessive GWG and optimise pregnancy outcomes.

Methods and design: The study is a two by two factorial randomised controlled demonstration trial conducted in Counties Manukau health region, New Zealand, a multi-ethnic region with a high prevalence of obesity. A total of 220 non-diabetic obese women with a singleton pregnancy will be recruited between $12^{0}$ and $17^{6}$ weeks. At recruitment, women are randomised to receive either a culturally tailored multifaceted dietary intervention or routine dietary advice, and either an oral probiotic or placebo capsule. Randomisation is undertaken via a web-based protocol, randomize.net, with a 1:1 ratio using stratification by body mass index (BMI) category (BMI of 30-34.9 or BMl $\geq 35 \mathrm{~kg} / \mathrm{m}^{2}$ ). The dietary intervention includes 4 customised nutrition education visits by a trained community health worker combined with motivational text messaging. Probiotic capsules consist of Lactobacillus rhamnosus GG and Bifidobacterium lactis BB12 at a dose of $7 \times 10^{9}$ colony-forming units one per day until birth. Probiotic and placebo capsules are identically pre-packed and labelled by a third party, and are prescribed in a double blinded fashion. Research assessments are conducted at enrolment, 28 weeks, 36 weeks, at birth and at 5 months post-delivery. The primary outcomes for the study are proportion of women with excessive GWG and infant birthweight.
\end{abstract}

Discussion: The HUMBA demonstration trial will assess the efficacy of a culturally tailored multifaceted dietary intervention and probiotic treatment in limiting excessive GWG and optimising birthweight in a multiethnic sample of obese pregnant women. If successful, either one or both of the interventions may be incorporated into future studies powered to investigate important pregnancy outcomes.

Trial registration: Australian New Zealand Clinical Trials Registry registration number: ACTRN12615000400561, Universal Trial Number: U1111-1155-0409. Date registered: 29 $9^{\text {th }}$ April 2015.

Keywords: Study protocol, Obesity, Nutritional intervention, Randomised controlled trial, Gestational weight gain, Probiotics, Birthweight

\footnotetext{
* Correspondence: I.mccowan@auckland.ac.nz

'Department of Obstetrics and Gynaecology, Faculty of Medical and Health

Science, University of Auckland, Private Bag 92019, Auckland, New Zealand

${ }^{2}$ South Auckland Clinical School, Faculty of Medical and Health Science, The

University of Auckland, Auckland, New Zealand

Full list of author information is available at the end of the article
} 


\section{Background}

The global obesity epidemic is of increasing concern, with 671 million people in the world currently estimated to be obese (body mass index $(\mathrm{BMI}) \geq 30 \mathrm{~kg} / \mathrm{m}^{2}$ ) [1]. Data from the United States suggests that without effective interventions, the rise in obesity will soon lead to reduction of lifeexpectancy in high-income countries [2]. New Zealand was rated the third most obese nation in the Organisation for Economic Co-operation and Development countries in 2014 [3]. The latest New Zealand national health survey showed that about 30\% of women at child-bearing age have a BMI of 30 or more, and the trend is increasing [4]. Of specific concern, the obesity rates are double in Māori and Pacific adults and children compared with those from European/other ethnic backgrounds [4], which highlights ethnic inequalities in the obesity epidemic in New Zealand.

Obese pregnant women have increased rates of most pregnancy complications including gestational diabetes (GDM), pre-eclampsia, caesarean section, and postpartum haemorrhage [5]. Their infants are at higher risk of congenital abnormalities, stillbirth, being born large for gestational age (LGA) and consequential traumatic birth and asphyxia [6,7]. They are also at increased risk of a range of neonatal complications, including respiratory problems, sepsis, seizures, hypoglycaemia and feeding difficulties [8]. In the long-term, maternal obesity has been associated with lower offspring cognitive function and increased risk of attention deficit hyperactivity and other psychiatric disorders [9, 10]. Moreover, maternal obesity is associated with adverse cardiometabolic health in the offspring. Fetal exposure to excessive nutrients such as maternal hyperlipidaemia and hyperglycaemia, can result in accelerated growth, especially of adipose tissue [7]. Larger infants with increased fat mass are more likely to become obese children [11], and are predisposed to high blood pressure, type 2 diabetes and other metabolic dysfunctions in adulthood [12, 13]. This creates a vicious intergenerational cycle, termed "developmental overnutrition" [14], which may have contributed to the increases in obesity and type 2 diabetes observed over the recent decades.

Based on a number of observational studies, the Institute of Medicine recommends 5-9 kg of gestational weight gain (GWG), in obese women, for optimal maternal and infant outcomes [15]. Exceeding recommended GWG is associated with increased risk of GDM, preeclampsia, LGA, and caesarean in labour independent of maternal BMI [16, 17]. Mothers with excessive weight gain are less likely to lose weight between pregnancies, and may enter further pregnancies even more overweight $[18,19]$. Further, excessive GWG compounds the associations between maternal obesity and offspring metabolic dysfunction and cognitive problems [9, 20, 21]. However, implementation of GWG guidelines in practice is challenging. Studies suggest that obese women are more likely to gain an excessive amount of weight during pregnancy than non-obese women [22], and may require more theoreticallydesigned interventions [23]. There is also an emerging demand from prenatal care providers for effective and reproducible intervention guidance [24].

Lifestyle interventions during pregnancy may limit GWG, however, limited data are available to identify the key components of intervention(s) that are responsible for the positive outcomes [23]. A systematic review reported that amongst diet, physical activity, and mixed approach interventions, dietary interventions were associated with the largest reduction in GWG (4 kg on average) and also with improved pregnancy outcomes [25]. A UK pilot trial of intensive behavioural intervention in obese pregnant women suggested that there is greater potential for change in dietary intake than in physical activity [26]. However, it has been consistently reported from several recent randomised controlled trials (RCT) that lifestyle interventions alone have a limited effect on reducing GWG in obese pregnant women [27-29]. Additional interventions in combination with lifestyle interventions therefore require evaluation in clinical trials to determine whether such combinations can achieve improvements in GWG.

Mobile phone texting technologies are increasingly being used to assist with weight loss. A systematic review has shown that use of mobile phone technologies in non-pregnant populations can result in successful weight loss with increased physical activity and improved nutrition [30]. There was a high level of acceptability and user satisfaction rating, with a significant number of participants stating they would recommend text messaging as a primary intervention to others [30]. A New Zealand multi-ethnic study, in an obese non-pregnant cohort, confirmed the feasibility of using mobile phone technology together with behaviour change techniques. They reported successful weight loss in participants [31]. A recent pilot study of 35 overweight and obese pregnant women reported a $2.7 \mathrm{~kg}$ mean GWG reduction with a texting intervention [32].

Modification of the gut microbiome by ingestion of probiotics is a novel pathway for possible intervention to prevent metabolic disease. The microbiome influences energy extraction from food [33], and satiety, inflammation, and glucose and lipid metabolism [34-36], with potential to reduce obesity and type 2 diabetes [37]. Probiotics are safe in pregnancy $[38,39]$ and provide a simple intervention in pill form. A randomised controlled trial of probiotics/placebo (Lactobacillus rhamnosus GG and Bifidobacterium lactis at $10^{10}$ colony-forming units/day) and nutritional advice in pregnant women in Finland showed an over $60 \%$ reduction in GDM, with a prevalence of $13 \%$ in the probiotic/nutrition group compared with $36 \%$ in placebo/ 
nutrition group and 34\% in controls [40]. In addition, a reduction in maternal central adiposity at 6 months postpartum and a $127 \mathrm{~g}$ average reduction of birthweight were demonstrated in the probiotic treatment group [41, 42]. Besides the reported efficacy of these probiotic capsule in reducing GDM, daily probiotic yoghurt has been associated with a $40 \%$ reduction in preeclampsia [43] which is also a common complication in obese pregnant women. A RCT of probiotics/placebo aimed to prevent GDM in overweight and obese women has started in Brisbane, Australia [44], but no studies have been reported to date, confined to obese pregnant women or in combination with intensive dietary intervention, aimed to prevent excessive GWG.

Pregnancy is described as a "teachable moment" providing a finite window during which women are more likely to undergo behavioural change if there are perceived benefits for their offspring [45]. Accordingly, we are undertaking an innovative, randomised controlled demonstration trial of probiotics or placebo plus an intensive, culturally tailored multifaceted dietary intervention in obese pregnant women in South Auckland, New Zealand. We hypothesise that in obese pregnant women the interventions will reduce: 1) the incidence of excessive GWG; and/or 2) infant birthweight.

\section{Methods and design}

\section{Study design and the setting}

The healthy mums and babies (HUMBA) study is a single centre two by two factorial randomised controlled demonstration trial (parallel groups) designed according to CONSORT guidelines [46] and the SPIRIT guidelines for preparation of protocols [47]. We aim to investigate whether or not an oral probiotic capsule consisting of Lactobacillus rhamnosus GG and Bifidobacterium lactis $B B 12$ at a dose of $7 \times 10^{9}$ colony-forming units per day each, or a multifaceted dietary intervention, can reduce excessive GWG and optimise infant birthweight in obese pregnant women.

The HUMBA study is being conducted in the multi-ethic Counties Manukau Health $(\mathrm{CMH})$ region, South Auckland, New Zealand, where $40 \%$ of pregnant women are obese in early pregnancy [48]. The ethnic distribution of the birthing population $(>7000$ per annum) in $\mathrm{CMH}$ is $36 \%$ Pacific, $24 \%$ Māori, $17 \%$ Asian, and 23\% European/other [48]. There is high socioeconomic deprivation in the region with $53 \%$ of the women who birthed in the area in 2013 categorised as being in the lowest socioeconomic quintile for New Zealand [48]. The perinatal mortality is higher in $\mathrm{CMH}$ than in any other district health board in the country [49]. The external review of maternity care in $\mathrm{CMH}$ has iterated obesity as a major risk factor for stillbirth in the Pacific community [49]. There is an urgent need for effective and sustainable interventions in this high-risk population to improve maternal and child health.

\section{Inclusion criteria}

Women with a singleton pregnancy, BMI $\geq 30 \mathrm{~kg} / \mathrm{m}^{2}$, between $12^{0}$ and $17^{6}$ weeks of gestation and able to provide informed written consent.

\section{Exclusion criteria}

Pre-existing diabetes or HbAlc at booking $\geq 50 \mathrm{mmol} / \mathrm{mol}$ [50], taking probiotic supplements, known congenital abnormality, medications or medical conditions which alter glucose metabolism, multiple pregnancy, bariatric surgery, and severe hyperemesis.

\section{Randomisation}

Randomisation is undertaken using a web-based protocol, randomize.net (http://randomize.net), using random block sizes (minimum 4; maximum 8). For randomisation purposes, each research midwife will serve as a proxy for 'clinical site' (this will enable each research midwife to be able to dispense the randomised study capsules at recruitment). Participants are stratified by 'clinical site' $(\mathrm{n}=2)$ and BMI category (BMI of 30-34.9 or BMI $\geq 35 \mathrm{~kg} / \mathrm{m}^{2}$ ) and randomly allocated in a 1:1 ratio to dietary intervention or routine dietary advice; and 1:1 ratio to probiotic or placebo (Fig. 1).

\section{Study interventions}

1. Dietary intervention vs routine dietary advice

1.1 Dietary intervention

The multifaceted dietary intervention comprises the following four components.

a) Encounters with nutrition advisor

The nutrition advisors are community health workers $(\mathrm{CHW})$, usually of Pacific or Māori ethnic background, and are experienced in engaging hardto-reach women in maternity care in our target population. CHW are an integral part of the health workforce in Counties Manukau. They have been trained in the Pacific Heart Beat Certificate of Nutrition by the New Zealand National Heart Foundation, which covers general nutrition as well as complementary extra training in nutrition during pregnancy [51]. A New Zealand registered dietitian has developed a standard operating procedure manual for the CHWs, and provides support, advice and oversight of the nutritional education package administered to participants by the CHWs. 


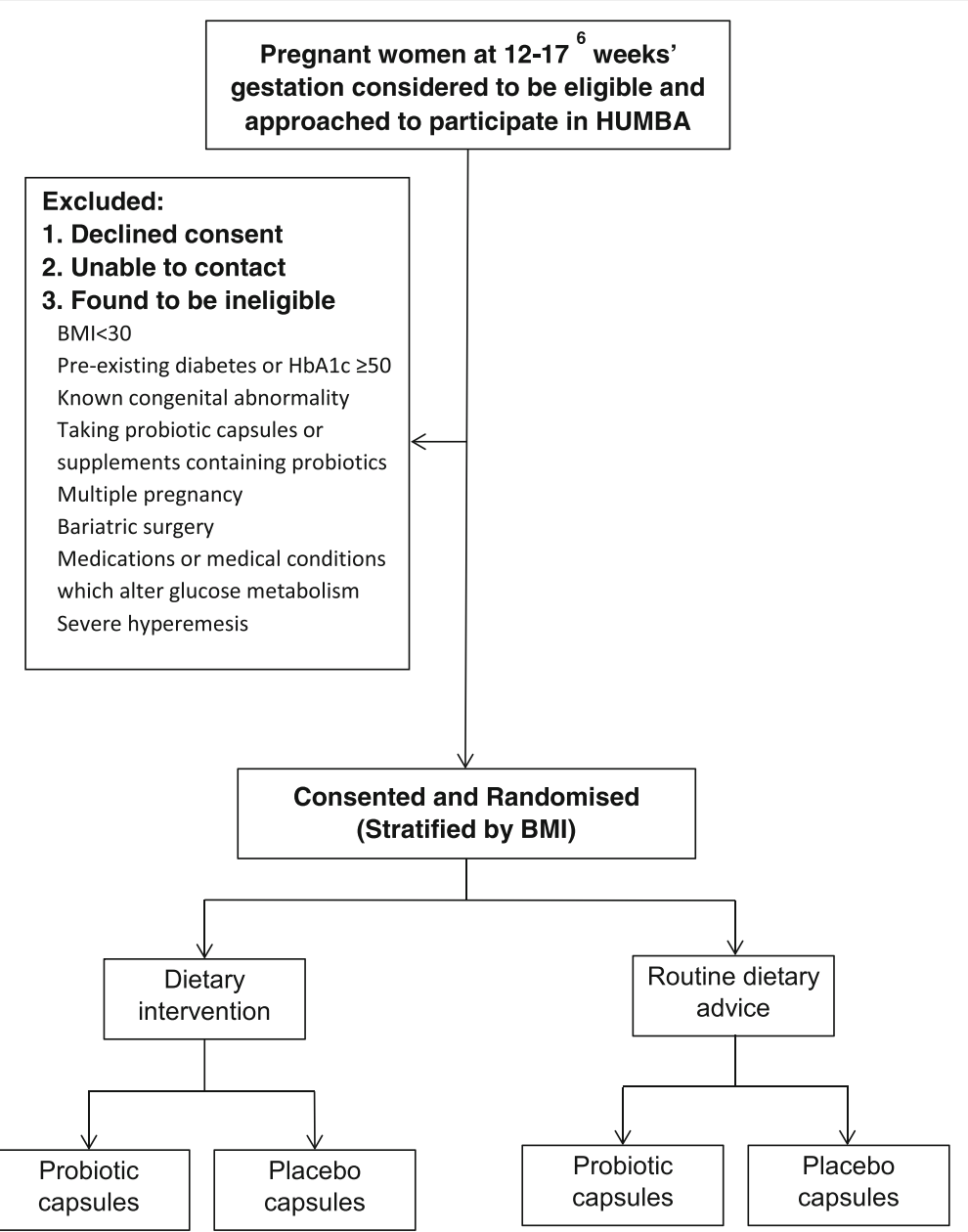

Fig. 1 Overview of recruitment and randomisation in the HUMBA study

The encounters include taking an initial brief diet and physical activity history followed by education on appropriate weight gain and in-depth education on healthy eating. The dietary education includes advice about: portion control, healthy food and drink choices, limiting energy dense foods, healthy recipes, label reading and managing cravings. Each participant has an initial 1-1.5 h educational session (on average at about 14 weeks' gestation) with the CHW. Three further 30-60 min face-to-face sessions are planned with the $\mathrm{CHW}$ at two weekly intervals and be completed by 28 weeks. Compliance with the dietary intervention is being assessed by the number of educational sessions participants attend with the CHW.

A HUMBA participant handbook containing detailed information about the suggested content of each of the four dietary education encounter visits is provided to women randomised to the dietary intervention. Participants are also encouraged to use the healthy recipes provided in their handbook.

b) Behaviour change techniques

CHWs have received training in evaluation methodology and counselling techniques, including healthy conversations [52]. Behaviour change techniques are incorporated in the nutrition education sessions including identifying barriers, self-monitoring, goal setting, and providing regular feedback [53]. Targets will be set for optimal GWG at the first visit with the CHW. Weight is measured and plotted on a personalised GWG chart, in the participant handbook, at each subsequent encounter.

c) Physical activity advice Physical activity advice which is included in the dietary intervention incorporates 
information from the Te Wai o Rona program (http://www.sportwaikato.org.nz/resourceslibrary.aspx? resource $=$ document-resourcecategory-2) [54] and the Royal College of Obstetricians and Gynaecologists (RCOG) guidelines for recreational exercise in pregnancy [55]. In Te Wai o Rona, there are four key physical activity messages which are relevant to pregnant women: 1) look for ways to be active every day, 2) increase daily exercise, 3 ) move more, add more steps, and 4) reduce sedentary leisure time. The RCOG guideline recommends that previously sedentary women begin with 15 min of exercise focusing on walking three times weekly and gradually increasing to 30 min sessions four times a week to daily [55].

d) Motivational texting Three times weekly motivational texting, which reinforces the educational content covered in the face-to-face meetings with the CHW, is implemented for those participants in the dietary intervention with cell phones (in our recent survey of pregnant women at $\mathrm{CMH}, 98 \%$ had a cell phone [56]). Text messaging continues until birth and incorporate messages about diet and physical activity.

\subsection{Routine dietary advice}

These women receive current best practice advice including a pamphlet produced by the New Zealand Ministry of Health (Eating for Healthy Pregnant Women) which contains dietary advice that follows current New Zealand nutrition guidelines [57]. They also receive a pamphlet providing information about healthy weight gain and physical activity in pregnancy [58].

\section{Probiotic/placebo intervention}

Probiotic or placebo capsules are taken once daily from enrolment between $12^{0}$ and $17^{6}$ weeks of gestation until birth.

\subsection{Probiotic intervention}

Participants randomised to probiotic treatment will receive probiotic capsules containing Lactobacillus rhamnosus GG and Bifidobacterium lactis BB12 (Chr. Hansen A/S, Horsholm, Denmark) at a dose of $7 \times 109$ colony-forming units per day each. Chr. Hansen A/S make the Probio-TecÖ BG-Vcap-6.5 capsules from library colonies which are meticulously DNA fingerprinted to ascertain the presence of Bifidobacterium BB12 and Lactobacillus rhamnosus GG only. The packaging and storage of the probiotics will comply with company specifications ensuring the quality of the product. This is the equivalent probiotic combination that was used by Luoto et al. in the Finnish study [40] and also being used in the ongoing study in Queensland [44].

\subsection{Placebo}

Participants randomised to placebo will receive identical capsules containing microcrystalline cellulose and dextrose anhydrate, also supplied by Chr. Hansen A/S, Horsholm, Denmark.

\section{Allocation concealment and blinding}

Christian Hansen has provided identically packaged canisters of placebo and probiotic capsules, containing 31 capsules each. AnQual Laboratories (School of Pharmacy, University of Auckland) have labelled the canisters using a pre-allocated random list. The kit list used to label the canisters was generated by the Project Manager and AnQual, using the Excel random function. This list has secure password protection and is stored with AnQual. The Project manager is the only HUMBA staff member with access to the probiotic/placebo allocation. Although it will not be possible for clinical and research staff to be blinded to the dietary intervention allocation, the key health outcomes including GWG, infant birthweight and results from the oral glucose tolerance test (OGTT), are not subject to bias.

\section{Assessment of compliance}

Compliance with the dietary intervention will be assessed by the number of educational sessions participants attend with the CHW. Compliance with probiotic/placebo will be assessed by the research team via participant self-report at 28 weeks, 36 weeks and birth visits.

\section{Engagement for recruitment}

We have a multi-pronged approach to optimise recruitment including through lead maternity caregivers (selfemployed midwives), community antenatal clinics, general practitioners, practice nurses, ultrasound clinics, community contacts, and social media. Our research team provides educational sessions for the health care providers to maximise reach for recruitment. Lead maternity caregivers notify the research team about contact details of eligible women who are interested in participating. The research midwife arranges a time to meet the woman in a suitable location to explain the study, confirm eligibility and obtain informed consent.

In order to exclude women with previously unrecognised Type 2 diabetes, HbA1c is measured in all participants (using the Roche cobas b 101 point-of-care system) prior to randomisation. If $\mathrm{HbA} 1 \mathrm{c}$ is $\geq 50 \mathrm{mmol} / \mathrm{mol}$, 


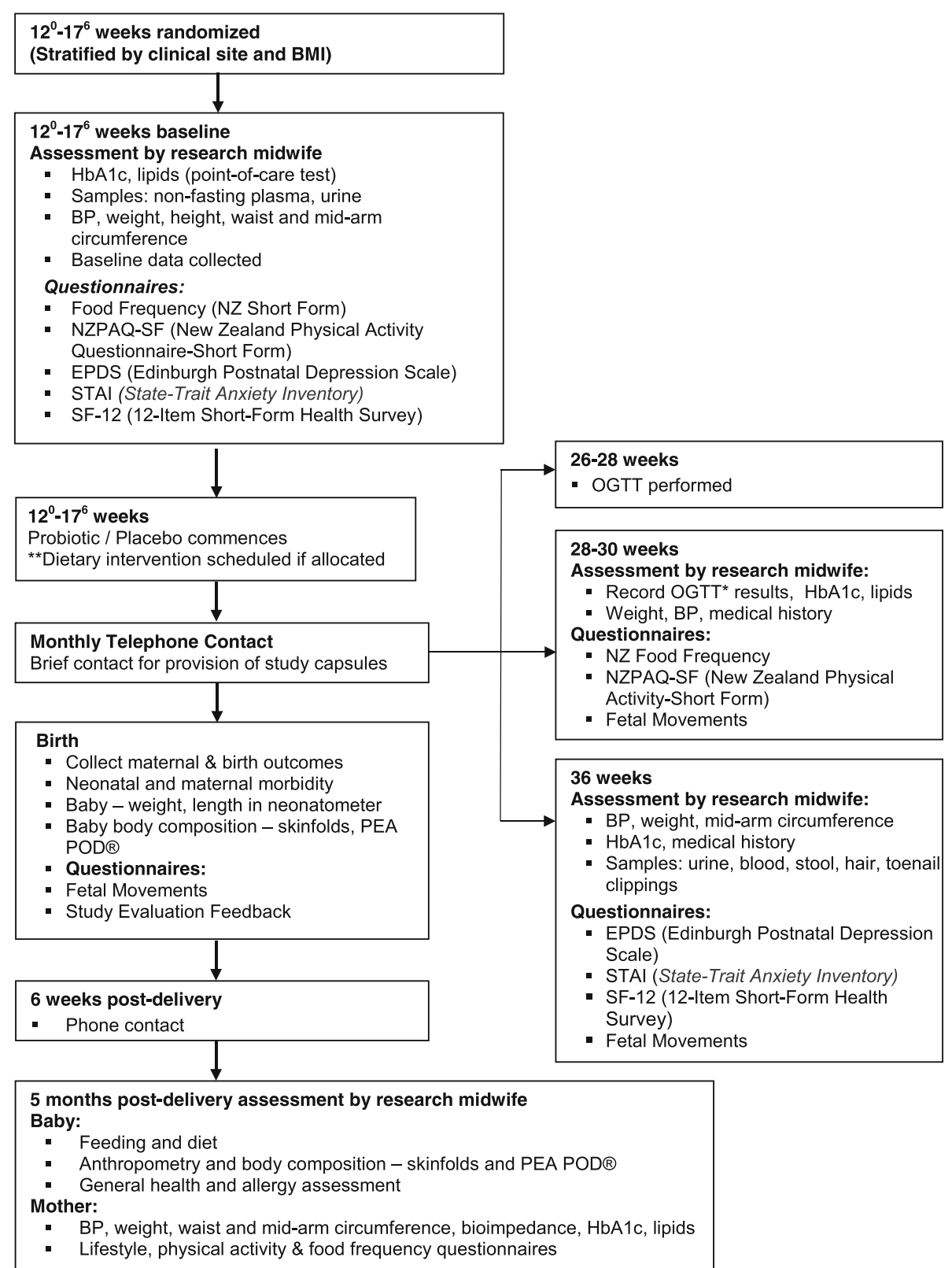

Fig. 2 Research plan flow chart for the HUMBA study. * Fasting, one-hour and two-hour glucose after $75 \mathrm{~g}$ glucose load. ** Women randomised to the dietary intervention will be seen 4 times at 2-3 weekly intervals between recruitment and the 26-28 week OGTT

women are ineligible and considered to have undiagnosed diabetes [50]. They will be referred to the Diabetes in Pregnancy Service at CMH. If the HUMBA point of care $\mathrm{HbA} 1 \mathrm{c}$ is $<50 \mathrm{mmol} / \mathrm{mol}$, then the result is not revealed to health practitioners.

\section{Measures/data collection}

Members of the research team will meet participants at pre-specified intervals during pregnancy (Fig. 2) to collect outcome data, provide further supplies of probiotic/placebo and check compliance.
Minimal data are collected with verbal consent in referred eligible women who decline to participate in the study. These minimal data include self-reported weight, height, age, ethnicity and reason for their nonparticipation.

\section{1st assessment visit $\left(12^{0}-17^{+6}\right.$ weeks)}

Confirmation of eligibility, informed consent, and randomisation is conducted at the first assessment visit. Comprehensive information is obtained from the woman and her clinical records, including: demographic, 
socioeconomic, educational and employment data; medical history; obstetric history such as parity, method of conception, gestation and accuracy of estimated date of delivery, previous pregnancy complications (GDM, hypertensive disease, pre-term delivery, caesarean section); family history of diabetes, hypertension and cardiovascular disease; history of smoking, alcohol and other drug use; medications and nutritional supplements; probiotic food ingestion; maternal anthropometrics: weight, height, waist and mid-arm circumference; blood pressure (BP); finger-prick blood lipid testing (Roche cobas b 101 point-of-care system); samples collection : non-fasting blood and urine specimen for biobank; questionnaires including: New Zealand Food Frequency Questionnaire - Short Form [59], New Zealand Physical Activity Questionnaire [60], Edinburgh Postnatal Depression Scale (EPDS) [61], State-Trait Anxiety Inventory (STAI) [62], and the 12-item Short-Form Health Survey (SF-12) [63]. It takes approximately $30 \mathrm{~min}$ to complete these questionnaires.

\section{$2^{\text {nd }}$ assessment visit (28-30 weeks)}

All women in the HUMBA trial are requested to have an OGTT at 26-28 weeks. The second assessment visit is scheduled after the OGTT at approximately 28-30 weeks. Weight, mid-arm circumference and BP are measured. The food frequency, physical activity questionnaires and a fetal movement questionnaire are completed by the participant. The fetal movement questionnaire is adopted from a previous study [64]. An updated medical history is obtained by the research midwife. The OGTT results are reported to the research team with fasting, one- and two-hour results enabling a diagnosis of GDM by the International Association of Diabetes in Pregnancy Study Groups (IADPSG) criteria [65]. The maternity care provider receives fasting and two-hour glucose results from the OGTT as per usual clinical practice using the New Zealand Society for the Study of Diabetes (NZSSD) criteria [66]. Women diagnosed with GDM by the NZSSD criteria will be referred by their maternity care provider to the Diabetes in Pregnancy Service (as per usual practice) and managed according to local guidelines including postpartum testing.

HbA1c and lipid concentrations are measured by finger prick using the Roche cobas b 101 point-of-care system.

\section{$3^{\text {rd }}$ assessment visit (36 weeks)}

Weight, mid-arm circumference, BP, and HbA1c and lipid concentrations (Roche cobas b 101 point-of-care system) are measured. Any pregnancy complications to date and use of antibiotics are recorded. The fetal movement questionnaire and functional health and well-being questionnaires including EPDS, STAI and SF-12 are repeated. Maternal urine, blood, stool, toenail clippings and hair samples are collected from consenting women for future molecular biology studies.

\section{$4^{\text {th }}$ assessment visit/post-delivery evaluation}

Maternal and neonatal outcomes are collected within $72 \mathrm{~h}$ of birth by a research midwife. Infants are weighed at birth (Mobile electronic baby scales, SECA 334, Germany) and detailed anthropometric measurements obtained by research midwives within $72 \mathrm{~h}$ of birth including crown-heel length by neonatometer, head, left mid-arm, chest and abdominal circumferences using a lasso tape, and subscapular, triceps and supra-iliac skinfolds by Harpenden calipers (average of 2 measurements, or median of 3 if initial measurements differ by $>0.4 \mathrm{~mm}$ ) [67]. Among consenting participants, infant body compositions are measured by air displacement plethysmography (PEA POD ${ }^{\circ}$ Cosmed, Illinois, USA) as soon as practical after birth. In these infants, anthropometry is performed at the same time as the PEA $\mathrm{POD}^{\circ}$ measurement. The rest of the birth outcomes are collected from the mothers and the babies' clinical records.

A short survey is administered to obtain feedback about participation in HUMBA and the study interventions, and the fetal movement questionnaire is repeated.

\section{$5^{\text {th }}$ assessment visit (5 months postpartum)}

A follow up appointment with the research midwife and a paediatrician are scheduled at 5 months ( \pm 2 weeks) postpartum. Maternal lifestyle and food frequency questionnaires, and infant health and well-being questionnaires are completed. Measurements of maternal BP, weight, height, waist and mid-arm circumference, and body composition by Bioelectrical Impedance Analysis [68] (ImpSFB7 ${ }^{\circ}$, ImpediMed, Brisbane, Australia) are undertaken. Data are collected on infant feeding, allergies, health and anthropometry (weight and length; head, left mid-arm, chest and abdominal circumference; subscapular, triceps and suprailiac skinfolds), and body composition by $\mathrm{PEA} \mathrm{POD}^{\circ}$, if consented. Infant feeding behaviours are assessed using the Baby Eating Behaviour Questionnaire (BEBQ) [69], and infant eating patterns and nutritional intake will be determined by a food frequency questionnaire. Timing of assessment will be based on corrected age. Consent will be obtained for ongoing contact when funding is obtained for further follow up.

\section{Primary outcomes}

The primary maternal outcome is the proportion of women with excessive GWG, defined as mean weekly weight gain $>0.22 \mathrm{~kg}$ between recruitment and 36 weeks 
(or weight at the closest gestation to 36 weeks' if 36 week weight is unavailable) [15].

The primary infant outcome is infant birthweight.

\section{Secondary outcomes}

Maternal secondary outcomes include:

- Maternal pregnancy glucose metabolism as assessed by OGTT parameters at 26-28 weeks [70], and $\mathrm{HbA} 1 \mathrm{c}$ at 28 and 36 weeks and 5 months postpartum.

- Changes in diet quality and dietary patterns between recruitment, 28-30 weeks and 5 months postpartum [59]

- Functional health and well-being (SF-12) at 36 weeks and 5 months postpartum [63]

- Depression and anxiety scores at 36 weeks' and 5 months postpartum $[62,63]$

- Maternal adiposity at 5 months postpartum (assessed by weight, BMI, waist and arm circumference and fat mass measured by bioimpedance)

- GDM by NZSSD criteria [66]

- Pregnancy induced hypertension (preeclampsia and gestational hypertension) [71]

- Mode of birth

- Blood lipid concentrations at 28-30 and 36 weeks' gestation and 5 months postpartum

- Maternal feedback about participation in the study (survey at post birth visit)

Infant secondary outcomes include:

- Neonatal anthropometry: head circumference and length, and associated Z-scores [72]; birthweight adjusted for length; girths (chest, arm and abdominal) adjusted for length; subscapular, triceps and suprailiac skin fold thickness adjusted for length; and arm muscle area adjusted for arm length

- Neonatal body composition (via PEA POD ${ }^{\circ}$ ) including fat mass and lean mass adjusted for length and fat mass adjusted for lean mass

- Gestational age at birth

- LGA, by customised [73] and population [72] references.

- Small for gestational age (SGA, $<10^{\text {th }}$ centile), by customised [73] and population [72] references.

- Admission to neonatal care unit (and reason)

- Neonatal composite morbidity, including birth trauma (fracture, brachial plexus injury, cephalohaematoma, subgaleal haematoma), hypoxic ischaemic encephalopathy, sepsis, respiratory distress requiring continuous positive airway pressure support, hypoglycaemia requiring dextrose treatment (buccal or intravenous)
- Initiation and establishment of breast feeding, including feeding in first two postnatal weeks (collected by phone call at 6 weeks and questionnaires)

- Infant anthropometry and body composition at 5 months of age, as detailed above

- Infant feeding over first 5 months (breast feeding, formula use, complementary feeding with solids)

- Feeding behaviour as assessed by BEBQ scores [69]

- Infant Nutritional intake at 5 months, estimated from a four day food frequency questionnaire

Other secondary outcomes include:

- Attendance at study visits

- Adherence to probiotic/placebo regime

- Cost effectiveness of the intervention

\section{Sample size and power calculation}

A total of 220 participants will be recruited. With $80 \%$ power and 100 subjects remaining in each main intervention group (allowing 10\% lost to follow-up) we can detect: $25 \%$ reduction in excessive GWG from $80 \%$ to $60 \%$ (based on an $80 \%$ rate of excess weight gain in obese participants in the SCOPE study) [16] and $227 \mathrm{~g}$ difference in mean birthweight (based on $\mathrm{CMH}$ data; mean $=3,638, \mathrm{SD}=521$ ). To allow for the two primary outcomes an alpha of 0.025 has been used for the power calculations (Bonferroni approach).

\section{Statistical analysis plan}

Analyses will follow the principle of intention-to-treat. Participants will be analysed according to the assigned treatment group at randomisation. Statistical models will adjust for the key randomisation stratification variable, BMI at recruitment.

Binary endpoints will be analysed using logistic regression to estimate odds ratios for each of the interventions (dietary intervention and probiotics). Continuous outcomes will be modelled using generalised linear models to estimate any changes in outcomes with the interventions (dietary intervention and probiotics) compared to their respective control groups.

Primary analyses will report marginal effects for each randomised exposure, with adjustment for co-intervention, BMI at recruitment (randomisation stratification variable), ethnicity and sex (infant outcomes). We will also test for interactions between the main effects (primary outcomes only), though this pilot trial has been powered only for the main effects.

Sensitivity analyses will be carried out according to compliance with the study interventions (primary outcomes); specifically these will be: 
- Those women who reported taking their probiotics most of the time at all time points (forgot to take 1-3 per month, or greater than 1 per week but not most days at 28 weeks, 36 weeks and birth visits).

- Those that attended at least 3 out of the 4 dietary education sessions.

Analyses of secondary outcomes involving maternal data collected at recruitment, 36 weeks' and 5 months' will be analysed using mixed methods to allow for the repeated measures of these outcomes over time. Measures of infant body composition (whole-body fat and fat-free mass) will be adjusted by infant sex and ethnicity, and skinfold thickness and arm muscle area will additionally be adjusted for infant length. For birth data, analyses will be adjusted for gestational age at birth (weeks). We will explore for an interaction between intervention effect and infant sex.

For the primary outcomes a two-sided alpha level $<0.025$ will indicate statistical significance (Bonferroni adjustment). For secondary outcomes, a two-sided alpha level $<0.05$ will indicate statistical significance.

Depending on the proportion and the pattern of missing data, multiple imputation may be used to impute missing data for some exploratory variables (secondary outcomes).

\section{Safety monitoring}

The research team will oversee and manage the project. A Trial Steering Committee has been established that contains a representative group of the named investigators who are responsible for the conduct of the trial and will follow CONSORT guidelines [46]. An independent committee (with no conflict of interest) has been appointed with established terms of reference to serve as the Data Safety and Monitoring Committee (DSMC). The DSMC includes experts in the fields of obstetrics, neonatology and epidemiology/statistics with experience in perinatal trials. They will assess the progress of the trial and review reports of serious adverse events and adverse events.

Serious adverse events are defined as maternal death, maternal admission to intensive care unit, fetal death, neonatal death or death up to primary hospital discharge, stage 2-3 neonatal encephalopathy, and any other serious adverse event that the principal or local lead investigator believes should be referred for independent review by the DSMC.

The DSMC will classify serious adverse events as to the likelihood of a causative association with the study intervention: no, unlikely, possible, or probable. The DSMC can request unblinding if there is any safety consideration related to the probiotic/placebo intervention.

\section{Data management}

Data will be collected in a timely fashion and entered directly into the web-based REDCap ${ }^{\text {Ts }}$ [74] study database using mobile tablets. Data are actively monitored by a data monitor following the Data Resolution Workflow produced by REDCap ${ }^{\text {th }}$ [74]. Electronic case record forms (CRFs) will be identified only by a study identification number (ID), with consent forms and identifying information stored in a separate, restricted database. In rare cases, when paper CRFs are used they will be coded using the study ID number, ensuring confidentiality of participants. All paper CRFs will be stored securely in locked cabinets for 10 years and access to all electronic data will be password protected and restricted to researchers directly involved with the study.

No interim analysis is planned.

\section{Discussion}

This two by two factorial design randomised controlled demonstration trial aims to reduce excessive GWG and optimise infant birthweight in a multi-ethnic sample of obese pregnant women. This RCT will inform whether a multifaceted dietary intervention and or probiotic treatment, are feasible interventions for obese pregnant women in the muti-ethnic $\mathrm{CMH}$ region. If successful, either one or both of the interventions are applicable to future clinical practice. It may also inform the design of a larger-scale intervention study for obese pregnant women that is powered to investigate pregnancy complications.

\section{Dissemination policy}

We will publish the results of HUMBA in scientific journals and present the results at relevant conferences locally and internationally. Our publications will also be available on the HUMBA study website, and will be shared by email with participants who have consented to receive a copy of the findings. Our multidisciplinary team of investigators will disseminate research findings widely via their clinical networks, including giving seminars and presentations to health professionals and community groups. We will utilise newsletters of relevant professional bodies to further disseminate research findings. Findings from HUMBA will also be incorporated into the relevant Cochrane systematic reviews thereby increasing dissemination both locally and internationally.

\section{Abbreviations \\ BEBQ: Baby Eating Behaviour Questionnaire; BMI: Body mass index; BP: Blood pressure; CHW: Community health worker; $\mathrm{CMH}$ : Counties Manukau Health; CRF: Case report form; DSMC: Data safety and monitoring committee; EPDS: Edinburgh Postnatal Depression Scale; GDM: Gestational diabetes mellitus; GWG: Gestational weight gain; HUMBA: Healthy mums and babies; IADPSG: International Association of the Diabetes and Pregnancy Study Groups; LGA: Large for gestational age; NZSSD: New Zealand Society for the Study of Diabetes; OGTT: Oral glucose tolerance test; RCOG: Royal College of Obstetricians and Gynaecologists; RCT: Randomised controlled trial; SF-12: \\ 12-item Short-Form Health Survey; STAI: State-Trait Anxiety Inventory}

\section{Acknowledgements}

We would like to acknowledge in-kind support from Christian Hansen A/S for probiotics/placebo capsules, Roche Diagnostics International Ltd for 
$\mathrm{HbA} 1 \mathrm{c}$ and Lipid bedside testing, Cure Kids for Koha for participants, and National Heart Foundation for helping with developing text me ssages.

\section{Funding}

This study was funded by Counties Manukau Health, Cure Kids Grant 3556, Lottery Health Research 353084, RANZCOG Mercia Barnes Trust, Gravida National Centre for Growth and Development, and University of Auckland Faculty Development Research Fund and Reinvestment Fund. Funding sources had no role in study design, data collection, analysis, interpretation, writing the report, or decision to submit the paper for publication.

\section{Availability of data and materials}

Not applicable.

\section{Authors' contributions}

KOG, LMEMCC, RST, JMDT, CAC and CJDM conceived and designed the study. All authors significantly contributed to the development and implementation of the protocol. KOG drafted the manuscript. ML, RST, JMDT, CAC, CJDM and LMEMCC critically evaluated the manuscript. JMDT designed and wrote the statistical part of the study. All authors have read and approved the final manuscript. LMEMcC will have full access to the final dataset and take responsibility for the integrity of the data.

\section{Competing interests}

The authors declare that they have no competing interests.

\section{Consent for publication}

Not applicable.

\section{Ethics approval and consent to participate}

Ethics approval was obtained from the Southern Health and Disability Ethics Committee, New Zealand (14/STH/205). All participants complete written informed consent and are advised that they can withdraw from the study at any time.

\section{Author details}

'Department of Obstetrics and Gynaecology, Faculty of Medical and Health Science, University of Auckland, Private Bag 92019, Auckland, New Zealand. ${ }^{2}$ South Auckland Clinical School, Faculty of Medical and Health Science, The University of Auckland, Auckland, New Zealand. ${ }^{3}$ The Liggins Institute, The University of Auckland, Auckland, New Zealand. ${ }^{4}$ Department of Paediatrics, Child and Youth Health, Faculty of Medical and Health Sciences, The University of Auckland, Auckland, New Zealand.

Received: 15 January 2016 Accepted: 8 November 2016 Published online: 24 November 2016

\section{References}

1. Ng M, Fleming T, Robinson M, Thomson B, Graetz N, Margono C, et al. Global, regional, and national prevalence of overweight and obesity in children and adults during 1980-2013: a systematic analysis for the Global Burden of Disease Study 2013. Lancet. 2014;384(9945):766-81.

2. Olshansky SJ, Passaro DJ, Hershow RC, Layden J, Carnes BA, Brody J, et al. A potential decline in life expectancy in the United States in the 21st century. NEJM. 2005; 352(11):1138-45.

3. OECD. Obesity Update 2014. Paris: OECD; 2014. http://www.oecd.org/els/ health-systems/Obesity-Update-2014.pdf. Accessed 28 Dec 2014.

4. Ministry of Health. Annual Update of Key Results 2014/15: New Zealand Health Survey. Wellington: Ministry of Health; 2015.

5. Magann EF, Doherty DA, Sandlin AT, Chauhan SP, Morrison JC. The effects of an increasing gradient of maternal obesity on pregnancy outcomes. Aust N Z J Obstet Gynaecol. 2013;53(3):250-257.

6. Heslehurst N, Simpson H, Ells LJ, Rankin J, Wilkinson J, Lang R, et al. The impact of maternal BMI status on pregnancy outcomes with immediate short-term obstetric resource implications: a meta-analysis. Obes Rev. 2008; 9(6):635-83.

7. HAPO Study Cooperative Research Group. Hyperglycaemia and Adverse Pregnancy Outcome (HAPO) Study: associations with maternal body mass index. BJOG. 2010;117(5):575-584.

8. Blomberg M. Maternal obesity, mode of delivery, and neonatal outcome. Obstet Gynecol. 2013;122(1):50-5.
9. Huang L, Yu X, Keim S, Li L, Zhang L, Zhang J. Maternal prepregnancy obesity and child neurodevelopment in the Collaborative Perinatal Project. Int J Epidemiol. 2014;43(3):783-92.

10. Van Lieshout RJ, Taylor VH, Boyle MH. Pre-pregnancy and pregnancy obesity and neurodevelopmental outcomes in offspring: a systematic review. Obes Rev. 2011;12(5)::548-559.

11. Taveras EM, Rifas-Shiman SL, Belfort MB, Kleinman KP, Oken E, Gillman MW. Weight status in the first 6 months of life and obesity at 3 years of age. Pediatrics. 2009;123(4):1177-83.

12. Boney CM, Verma A, Tucker R, Vohr BR. Metabolic syndrome in childhood: association with birth weight, maternal obesity, and gestational diabetes mellitus. Pediatrics. 2005;115(3):e290-6.

13. Penfold NC, Ozanne SE. Developmental programming by maternal obesity in 2015: Outcomes, mechanisms, and potential interventions. Horm Behav. 2015:76:143-152

14. Dabelea D, Harrod CS. Role of developmental overnutrition in pediatric obesity and type 2 diabetes. Nutr Rev. 2013;71 Suppl 1:S62-7.

15. Rasmussen KM, Yaktine AL, editors. Weight Gain During Pregnancy: Reexamining the Guidelines. Washington: National Academies Press; 2009

16. Chung JG, Taylor RS, Thompson JM, Anderson NH, Dekker GA, Kenny LC, et al. Gestational weight gain and adverse pregnancy outcomes in a nulliparous cohort. Eur J Obstet Gynecol Reprod Biol. 2013;167(2):149-53.

17. Park S, Sappenfield WM, Bish C, Salihu H, Goodman D, Bensyl DM. Assessment of the Institute of Medicine recommendations for weight gain during pregnancy: Florida, 2004-2007. Matern Child Health J. 2011;15(3):289-301.

18. Crosby DA, Collins M, O'Higgins A, Mullaney L, Farah N, Turner MJ. Interpregnancy changes in maternal weight and body mass index. Am J Perinatol. 2015;30(2):199-204.

19. Siega-Riz AM, Viswanathan M, Moos MK, Deierlein A, Mumford S, Knaack J, et al. A systematic review of outcomes of maternal weight gain according to the Institute of Medicine recommendations: birthweight, fetal growth, and postpartum weight retention. Am J Obstet Gynecol. 2009;201(4):339 e331-314.

20. Perng W, Gillman MW, Mantzoros CS, Oken E. A prospective study of maternal prenatal weight and offspring cardiometabolic health in midchildhood. Ann Epidemiol. 2014;24(11):793-800 e791.

21. Hochner H, Friedlander Y, Calderon-Margalit R, Meiner V, Sagy Y, AvgilTsadok M et al. Associations of maternal prepregnancy body mass index and gestational weight gain with adult offspring cardiometabolic risk factors: the Jerusalem Perinatal Family Follow-up Study. Circulation. 2012:125(11):1381-9.

22. Kim SY, Sharma AJ, Sappenfield W, Wilson HG, Salihu HM. Association of maternal body mass index, excessive weight gain, and gestational diabetes mellitus with large-for-gestational-age births. Obstet Gynecol. 2014;123(4):737-44.

23. Brown MJ, Sinclair M, Liddle D, Hill AJ, Madden E, Stockdale J. A systematic review investigating healthy lifestyle interventions incorporating goal setting strategies for preventing excess gestational weight gain. PLOS ONE. 2012;7(7):e39503.

24. Stotland NE, Gilbert P, Bogetz A, Harper CC, Abrams B, Gerbert B. Preventing excessive weight gain in pregnancy: how do prenatal care providers approach counseling? J Womens Health. 2010;19(4):807-14.

25. Thangaratinam S, Rogozinska E, Jolly K, Glinkowski S, Roseboom T, Tomlinson JW, et al. Effects of interventions in pregnancy on maternal weight and obstetric outcomes: meta-analysis of randomised evidence. BMJ. 2012;344:e2088.

26. Poston L, Briley AL, Barr S, Bell R, Croker H, Coxon K, et al. Developing a complex intervention for diet and activity behaviour change in obese pregnant women (the UPBEAT trial); assessment of behavioural change and process evaluation in a pilot randomised controlled trial. BMC Pregnancy Childbirth. 2013;13:148

27. Poston $L$, Bell R, Croker H, Flynn AC, Godfrey KM, Goff $L$, et al. Effect of a behavioural intervention in obese pregnant women (the UPBEAT study): a multicentre, randomised controlled trial. Lancet Diabetes Endocrinol. 2015;3(10):767-77.

28. Dodd JM, MCPhee AJ, Turnbull D, Yelland LN, Deussen AR, Grivell RM, et al. The effects of antenatal dietary and lifestyle advice for women who are overweight or obese on neonatal health outcomes: the LIMIT randomised trial. BMC Med. 2014;12:163.

29. Vinter CA, Jensen DM, Ovesen P, Beck-Nielsen H, Jorgensen JS. The LiP (Lifestyle in Pregnancy) study: a randomized controlled trial of lifestyle intervention in 360 obese pregnant women. Diabetes Care. 2011;34(12):2502-7. 
30. Stephens J, Allen J. Mobile phone interventions to increase physical activity and reduce weight: a systematic review. J Cardiovasc Nurs. 2013;28(4):320-9.

31. Ni Mhurchu C, Whittaker R, McRobbie H, Ball K, Crawford D, Michie J, et al. Feasibility, acceptability and potential effectiveness of a mobile health (mHealth) weight management programme for New Zealand adults. BMC Obes. 2014;1:10

32. Pollak Kl, Alexander SC, Bennett G, Lyna P, Coffman CJ, Bilheimer A, et al. Weight-related SMS texts promoting appropriate pregnancy weight gain: a pilot study. Patient Educ Couns. 2014;97(2):256-60.

33. Turpin W, Humblot C, Thomas M, Guyot JP. Lactobacilli as multifaceted probiotics with poorly disclosed molecular mechanisms. Int J Food Microbiol. 2010;143(3):87-102.

34. Abd El-Gawad IE-SM, Hafez S, El-Zeini H, Saleh F. The hypocholesterolaemic effect of milk yoghurt and soy-yoghurt containing bifidobacteria in rats fed on a cholesterol-enriched diet. Int Dairy J. 2005;15(1):37-44.

35. Flint HJ. The impact of nutrition on the human microbiome. Nutr Rev. 2012;70 Suppl 1:S10-3.

36. Turnbaugh PJ, Ley RE, Mahowald MA, Magrini V, Mardis ER, Gordon JI. An obesity-associated gut microbiome with increased capacity for energy harvest. Nature. 2006:444(7122):1027-31.

37. Power S FG, O'Toole $\mathrm{P}$, et al. Metabolic syndrome and obesity in adults. In: Probiotic bacteria and their effect on human health and well-being. edn. Edited by A. Guarino EQ, WA Walker: Karge; 2013: 103-107.

38. Allen SJ, Jordan S, Storey M, Thornton CA, Gravenor M, Garaiova I, et al. Dietary supplementation with lactobacilli and bifidobacteria is well tolerated and not associated with adverse events during late pregnancy and early infancy. J Nutr. 2010;140(3):483-8.

39. Elias J, Bozzo P, Einarson A. Are probiotics safe for use during pregnancy and lactation? Can Fam Physician. 2011;57(3):299-301.

40. Luoto $R$, Laitinen $K$, Nermes M, Isolauri E. Impact of maternal probioticsupplemented dietary counselling on pregnancy outcome and prenatal and postnatal growth: a double-blind, placebo-controlled study. Br J Nutr. 2010; 103(12):1792-9.

41. IImonen J, Isolauri E, Poussa T, Laitinen K. Impact of dietary counselling and probiotic intervention on maternal anthropometric measurements during and after pregnancy: a randomized placebo-controlled trial. Clin Nutr. 2011; 30(2):156-64.

42. Barrett HL, Dekker Nitert M, Conwell LS, Callaway LK. Probiotics for preventing gestational diabetes. Cochrane Database Syst Rev. 2014;2:CD009951.

43. Brantsaeter AL, Myhre R, Haugen M, Myking S, Sengpiel V, Magnus P, et al. Intake of probiotic food and risk of preeclampsia in primiparous women: the Norwegian Mother and Child Cohort Study. Am J Epidemiol. 2011; 174(7):807-15.

44. Nitert MD, Barrett HL, Foxcroft K, Tremellen A, Wilkinson S, Lingwood B, et al. SPRING: an RCT study of probiotics in the prevention of gestational diabetes mellitus in overweight and obese women. BMC Pregnancy Childbirth. 2013;13:50.

45. Phelan S. Pregnancy: a "teachable moment" for weight control and obesity prevention. Am J Obstet Gynecol. 2010;202(2):135 e131-138.

46. Schulz KF, Altman DG, Moher D. CONSORT 2010 Statement: updated guidelines for reporting parallel group randomised trials. BMC Med. 2010;8:18.

47. Chan AW, Tetzlaff JM, Altman DG, Laupacis A, Gotzsche PC, Krleza-Jeric K, et al. SPIRIT 2013 statement: defining standard protocol items for clinical trials. Ann Intern Med. 2013;158(3):200-7.

48. Tout S, Mildenhall L, Thompson T, Ellis C, Stark L, Knetsch N. Maternity Quality and safety Programme Annual Report 2014-2015. Auckland: Counties Manukau Health; 2015. http://www.countiesmanukau.health.nz/ assets/About-CMH/Reports-and-planning/Maternity/2014-2015-MaternityQuality-Safety-Programme.pdf. Accessed 28 Dec 2015.

49. Paterson R, Candy A, Lilo S, McCowan L, Naden R, O'Brien M. External review of maternity care in the Counties Manukau District. Auckland: Counties Manukau Health; 2012. http://www.countiesmanukau.health.nz/ assets/About-CMH/Reports-and-planning/Maternity/2012-CMH-externalreport-maternity-care-review.pdf. Accessed 28 Dec 2015.

50. Braatvedt GD, Cundy T, Crooke M, Florkowski C, Mann Jl, Lunt H, et al. Understanding the new HbA1C units for the diagnosis of Type 2 diabetes. $\mathrm{N}$ Z Med J. 2012;125(1362):70

51. Certificate in Pacific Nutrition.http://www.heartfoundation.org.nz/ programmes-resources/pacific-health/pacific-healthy-eating/certificate-inpacific-nutrition. Accessed 28 Dec 2015
52. Barker M, Baird J, Lawrence W, Jarman M, Black C, Barnard K, et al. The Southampton Initiative for Health: a complex intervention to improve the diets and increase the physical activity levels of women from disadvantaged communities. J Health Psychol. 2011;16(1):178-91.

53. Gardner B, Wardle J, Poston L, Croker H. Changing diet and physical activity to reduce gestational weight gain: a meta-analysis. Obes Rev. 2011;12(7):e602-20.

54. Simmons D, Rush E, Crook N. Development and piloting of a community health worker-based intervention for the prevention of diabetes among New Zealand Maori in Te Wai o Rona: Diabetes Prevention Strategy. Public Health Nutr. 2008;11(12):1318-25.

55. Bell BB, Dooley P. Exercise in pregnancy: guidelines and audit committee of the Royal College of Obstetricians and Gynaecologists. In: RCOG Statement No 4; 2006:7.

56. Okesene-Gafa K, Chelimo C, Chua S, Henning M, McCowan L. Knowledge and beliefs about nutrition and physical activity during pregnancy in women from South Auckland region, New Zealand. Aust N Z J Obstet Gynaecol. 2016; 56(5):471-483. doi: 10.1111/ajo.12456.

57. Ministry of Health. Eating for healthy pregnant women. Wellington: Ministry of Health; 2013. http://www.health.govt.nz/publication/food-and-nutritionguidelines-healthy-pregnant-and-breastfeeding-women-background-paper. Accessed 28 Dec 2015.

58. Ministry of Health. Guidance for Healthy Weight Gain in Pregnancy. Welington: Ministry of Health; 2014. http://www.health.govt.nz/system/files/ documents/publications/guidance-for-healthy-weight-gain-in-pregnancyjun14-v2.pdf. Accessed 28 Dec 2015.

59. Sam CH, Skeaff S, Skidmore PM. A comprehensive FFQ developed for use in New Zealand adults: reliability and validity for nutrient intakes. Public Health Nutr. 2014:17(2):287-96.

60. McLean G, Tobias MI. The New Zealand Physical Activity Questionnaires: Report on the validation and use of the NZPAQ-LF and NZPAQ-SF selfreport physical activity survey instruments. Wellington: Sport and Recreation New Zealand (SPARC); 2004

61. Peindl KS, Wisner $\mathrm{KL}$, Hanusa $\mathrm{BH}$. Identifying depression in the first postpartum year: guidelines for office-based screening and referral. J Affect Disord. 2004;80(1):37-44.

62. Marteau TM, Bekker $\mathrm{H}$. The development of a six-item short-form of the state scale of the Spielberger State-Trait Anxiety Inventory (STAI). Br J Clin Psychol. 1992;31(Pt 3):301-6.

63. Gandek B, Ware JE, Aaronson NK, Apolone G, Bjorner JB, Brazier JE, et al. Cross-validation of item selection and scoring for the SF-12 Health Survey in nine countries: results from the IQOLA Project. International Quality of Life Assessment. J Clin Epidemiol. 1998;51(11):1171-8.

64. Stacey T, Thompson JM, Mitchell EA, Ekeroma AJ, Zuccollo JM, McCowan LM Maternal perception of fetal activity and late stillbirth risk: findings from the Auckland Stillbirth Study. Birth. 2011;38(4):311-6. doi: 10.1111/j.1523-536X.2011. 00490.x.

65. Metzger BE, Gabbe SG, Persson B, Buchanan TA, Catalano PA, Damm P, et al. International association of diabetes and pregnancy study groups recommendations on the diagnosis and classification of hyperglycemia in pregnancy. Diabetes Care. 2010;33(3):676-82.

66. Simmons D, Rowan J, Reid R, Campbell N. Screening, diagnosis and services for women with gestational diabetes mellitus (GDM) in New Zealand: a technical report from the National GDM Technical Working Party. N Z Med J. 2008;121(1270):74-86.

67. Catalano PM, Thomas AJ, Avallone DA, Amini SB. Anthropometric estimation of neonatal body composition. Am J Obstet Gynecol. 1995;173(4):1176-81.

68. Heitmann BL. Prediction of body water and fat in adult Danes from measurement of electrical impedance. A validation study. Int J Obes. 1990;14(9):789-802.

69. Llewellyn $\mathrm{CH}$, van Jaarsveld $\mathrm{CH}$, Johnson L, Carnell S, Wardle J. Development and factor structure of the Baby Eating Behaviour Questionnaire in the Gemini birth cohort. Appetite. 2011;57(2):388-96.

70. Kim S, Min WK, Chun S, Lee W, Chung HJ, Lee PR, Kim A. Quantitative risk estimation for large for gestational age using the area under the 100-g oral glucose tolerance test curve. J Clin Lab Anal. 2009;23(4):231-6.

71. Tranquilli AL, Dekker G, Magee L, Roberts J, Sibai BM, Steyn W, et al. The classification, diagnosis and management of the hypertensive disorders of pregnancy: A revised statement from the ISSHP. Pregnancy Hypertens. 2014; 4(2):97-104.

72. Cole TJ, Williams AF, Wright CM, RCPCH Growth Chart Expert Group. Revised birth centiles for weight, length and head circumference in the UKWHO growth charts. Ann Hum Biol. 2011;38(1):7-11. 
73. Anderson NH, Sadler LC, Stewart AW, Fyfe EM, McCowan LM. Independent risk factors for infants who are small for gestational age by customised birthweight centiles in a multi-ethnic New Zealand population. Aust N Z J Obstet Gynaecol. 2013;53(2):136-42

74. Harris PA, Taylor R, Thielke R, Payne J, Gonzalez N, Conde JG. Research electronic data capture (REDCap)-a metadata-driven methodology and workflow process for providing translational research informatics support. J Biomed Inform. 2009;42(2):377-81

Submit your next manuscript to BioMed Central and we will help you at every step:

- We accept pre-submission inquiries

- Our selector tool helps you to find the most relevant journal

- We provide round the clock customer support

- Convenient online submission

- Thorough peer review

- Inclusion in PubMed and all major indexing services

- Maximum visibility for your research

Submit your manuscript at www.biomedcentral.com/submit 